\title{
Nanoparticle Physical Characterization
}

National Cancer Institute

\section{Source}

National Cancer Institute. Nanoparticle Physical Characterization. NCI Thesaurus. Code C62362.

The measurement and description of information pertaining to the determination of the physical properties of nanoparticles. These analyses enable the quantification of thermal, electrical and mechanical parameters of these entities. 